\title{
Manipulative and Body-Based Intervention Procedure
}

National Cancer Institute

\section{Source}

National Cancer Institute. Manipulative and Body-Based Intervention Procedure. NCI

Thesaurus. Code C15907.

Procedures which rely upon movement and manipulation of the body.T hey focus

primarily on the structures and systems of the body, including the bones and joints, the soft tissues, and the circulatory and lymphatic systems 